\title{
A Case of an Incidentally Detected Retroperitoneal Schwannoma: Current Approaches to Diagnosis and Treatment of Schwannoma
}

\author{
Mehmet Onur Gul ${ }^{1}$ (D), Mehmet Torun Huseyin ${ }^{1}$ (D) Tahsin Gulseven $^{1}$ (D), Cebrail Akyuz ${ }^{2}$ (D) \\ ${ }^{1}$ Haydarpasa Educational and Research Hospital, General Surgery Clinics, Istanbul, Turkey \\ ${ }^{2}$ Haydarpasa Educational and Research Hospital, General Surgery and Gastroentorology Gastroenteroloji Clinics, Istanbul, Turkey \\ Correspondence Author: Mehmet Onur Gul \\ E-mail: mehmetonurgul@hotmail.com \\ Received: 24.04.2018 Accepted: 06.08.2018
}

\begin{abstract}
Schwannomas are tumors that originate from Schwann cells in the nerve sheath. They most commonly manifest in the head, neck and extremities and rarely can arise from the retroperitoneum. Here, we reported a case of an incidentally detected retroperitoneal schwannoma in a 34 -year-old female and discussed the current techniques for diagnosis and treatment methods of schwannomas.

Keywords: Schwannoma, Retroperitoneal, incidental
\end{abstract}

\section{INTRODUCTION}

Schwannomas are tumors originating from Schwann cells in the peripheral nerve sheath. Most of the Schwannomas originate from the peripheral nerves of the face, neck and extremity, nevertheless some of them rarely originate from the retroperitoneal region(1). Retroperitoneal schwannomas add up to $0.3-3.2 \%(2,3)$ of primer schwannomas and $0.3-6 \%$ of all retroperitoneal tumors $(4,5)$.

We aimed to investigate the diagnostic and therapeutic methods of schwannomas in the direction of a rare retroperitoneal schwannoma case.

\section{CASE REPORT}

In this study, the patient's informed consent was obtained that the pictures and patient information could be used in scientific content or articles.

No pathology was found on physical examination and routine laboratory examinations of a 34 year old female patient who was evaluated for complaints of dyspeptic ongoing for approximately 3 months. In abdominal ultrasonography performed to investigate the etiology of dyspepsia; a mass lesion of $24 \times 26 \mathrm{~mm}$ was found between the pancreas posterior and the aorta. Tumor markers (CEA, CA 19-9) were normal. In the computed tomography; the pancreas was closely related to the portal vein and splenic venous branch in the corpus inferior compartment but without significant invasion findings, adjacent to the superior mesenteric artery and celiac truncus. Pancreas was with smooth contour and could not be selected with clear boundaries. In the arterial phase no contrast enhancement was seen and in the venous phase a solid mass lesion with peripheral contrast enhancement was observed. In the histopathological evaluation of trucut biopsy material made with computerized tomography; it was thought to be the peripheral nerve sheath tumor of the anterior segment due to diffuse and strong S-100 positivity. Because of the hypocellular and proliferative activity of the lesion was low, the mass was evaluated as benign schwannoma. First thought to be a benign schwannoma when retroperitoneal mass described in patient with follow-up and treatment options; the patient preferred to remove the mass with surgery. There upon patient was operated and mass which located postero inferior pancreatic corpus was reached in operation. (Figure 1) Mass, which was not invasive but attached superior mesenteric artery, portal vein and splenic vein, was completely excised without severe haemorrhage (Figure 2). The patient was discharged without any problems on the 7th postoperative day.

In the histopathological examination of the material; the tumor is separated from the surrounding tissue by a uniform boundary and no significant cell atypia, no mitosis or necrosis were seen. The presence of perivascular hyalinization (Figure 3 ) as well as diffuse and strong immunoreactivity with S-100 (Figure 4) were detected. This case was diagnosed as benign. schwannoma. 


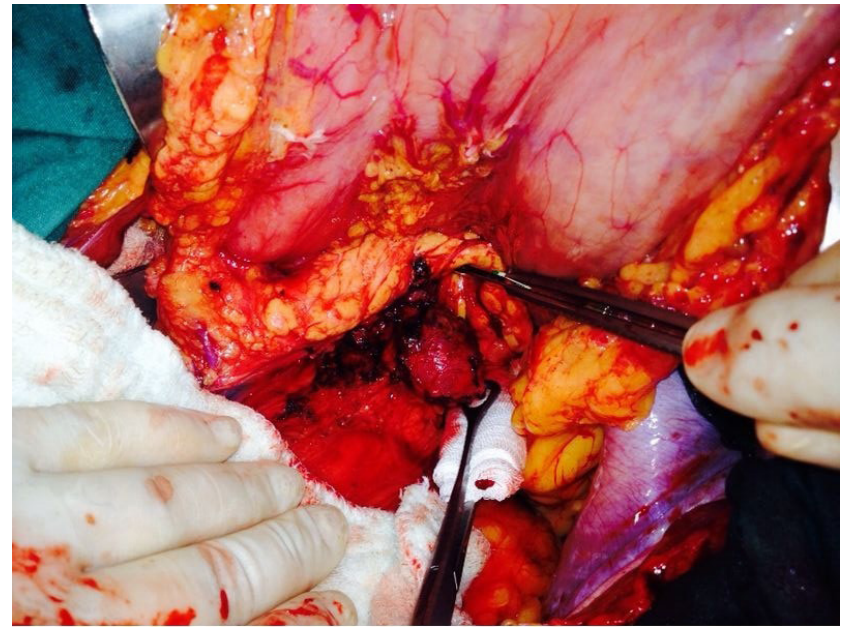

Figure 1. Postero-inferior mass of pancreas

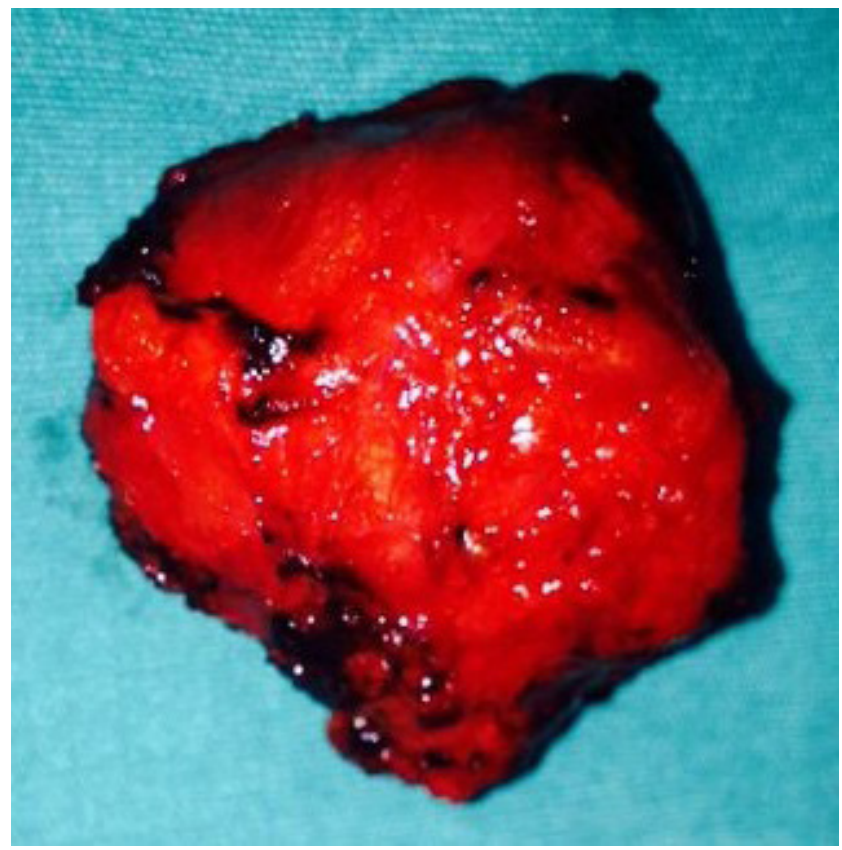

Figure 2. Fully excised, properly limited mass

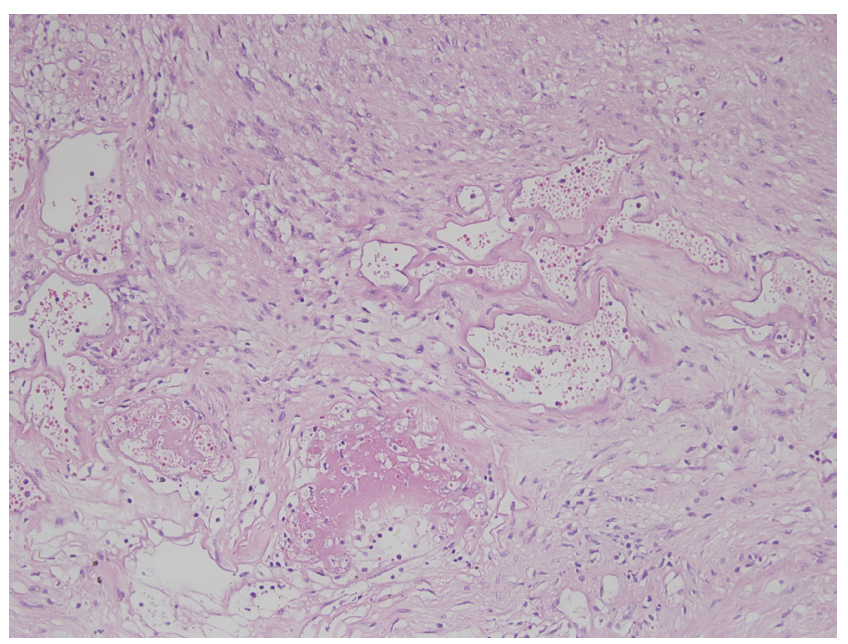

Figure 3. Perivascular hyelinization (HEx4O)

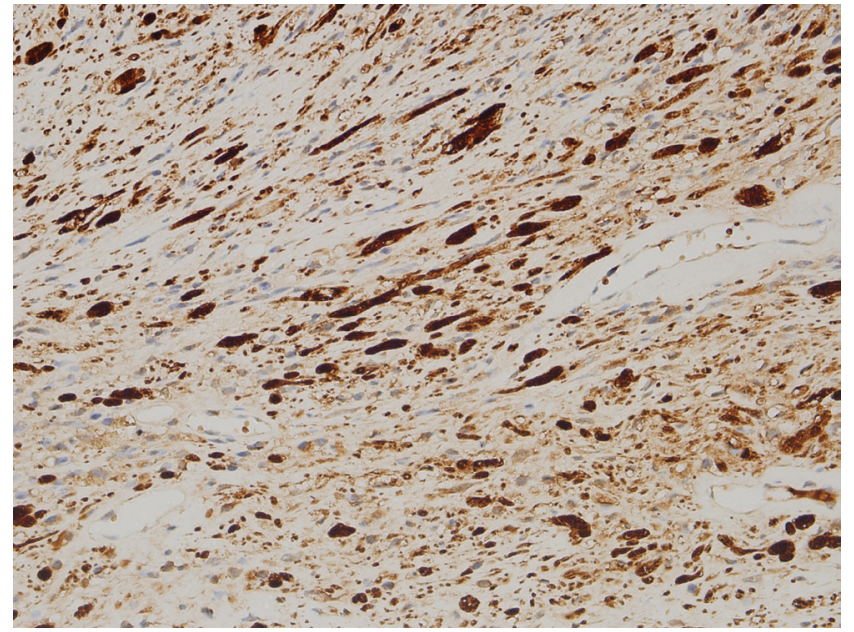

Figure 4. Widespread and strong nuclear staining with S-100

\section{DISCUSSION}

Since retroperitoneal region may be flexible, tumors developing in this area are not detected as symptom but incidentally detected without reaching large dimensions in general. Therefore, the diameter of the retroperitoneal Schwannomas causing the symptom is generally greater than $8 \mathrm{~cm}$ (1). While USG showed a hypoechoic mass with uniformly limited and minimal heterogeneous internal echoes in imaging, because of the low vascularity of the schwannomas, hypodense, well-defined mass is seen with contrast-enhanced CT (6). The definitive diagnosis can only be made if a strong S-100 positivity is detected after histopathological evaluation (7). In addition, the differentiation of benign or malignant schwannoma is important and necessary to determine the follow-up and treatment strategy.

There are few standard criteria for the diagnosis of malignant Schwannoma, and the size of the tumor is not sufficient to diagnose malignancy alone. First, the presence of histopathological criteria such as cellulite, nuclear atypia, and tumor necrosis increase point the possibility of malignancy (1). Especially in large schwannomas, secondary degenerative changes such as cystic degeneration, hemorrhage, necrosis and calcification can develop. In this case, imaging methods can not distinguish schwannomas from malignant retroperitoneal tumors $(8,9)$. For this reason, surgical removal of all large retroperitoneal masses is recommended. Malignant schwannomas are generally larger than $5 \mathrm{~cm}$ in size, with intimal bloody or necrosis, and high Ki 67 index (5-65\%), which are not well distinguished from surrounding tissues (10). The primary purpose of the treatment is to completely remove the cyst without causing damage to the nerve and causing severe haemorrhage. Benign Schwannomas do not invade vessels, but when benign tumors are large they may be involved in peripheral organs. In this case, the tumor is benign so more conservative treatment may be preferred, considering the morbidity results of surrounding tissue resection. However, 
complete resection with negative margin should be targeted to malignant Schwannomas (1). In addition, some authors have reported that asymptomatic, small retroperitoneal schwannomas without histopathological malignancy findings can be followed without surgery (6).

\section{REFERENCES}

[1] Li Q, Gao C, Juzi JT, Hao X. Analysis of 82 cases of retroperitoneal schwannoma. ANZ J Surg. 2007; 77(4): 237-240.

[2] Narasimha A, Kumar MH, Kalyani R, Madan M. Retroperitoneal cystic schwannoma: A case report with review of literature. J Cytol 2010; 27(4): 136-139.

[3] Theodosopoulos T, Stafyla VK, Tsiantoula P, Yiallourou A, Marinis A, Kondi-Pafitis, Chatziioannou A, Boviatsis E, Voros D. Special problems encountering surgical manage ment of large retroperitoneal schwannomas. World J Surg Oncol 2008 3; 6: 107.

[4] Misra MC, Bhattacharjee HK, Hemal AK, Bansal VK. Laparoscopic management of rare retroperitoneal tumors. Surg Laparosc Endosc Percutan Tech 2010 Jun; 20(3): e117122.
[5] Pinto D, Kaidar-Person O, Cho M, Zundel N, Szomstein S, Rosenthal RJ. Laparoscopic resection of a retroperitoneal degenerative schwannoma: a case report and review of the literature. Surg Laparosc Endosc Percutan Tech 2008; 18(1): 121-123.

[6] Kudo, Taiki, et al. "Three cases of retroperitoneal schwannoma diagnosed by EUS-FNA." World Journal of Gastroenterology: WJG 17.29 (2011): 3459.

[7] Trifero M, Frattaroli FM, Ciccone AM, Guadalaxara A, Oddi G, Pappalardo G. Retroperitoneal malignant schwannoma: A case report. Tumori 1994 30; 80(3): 238-240.

[8] Takatera H, Takiuchi H, Namiki M, Takaha M, Ohnishi S, Sonoda T. Retroperitoneal schwannoma. Urology 1986 ; 28(6): 529531.

[9] Daneshmand S, Youssefzadeh D, Chamie K, Boswell W, Wu N, Stein JP. Benign retroperitoneal schwannoma: a case series and review of the literature. Urology 2003; 62(6): 993-997.

[10] Woodruff JM, Kourea HP, Louis DN, Scheithauer BW. In: Kleihues P, Cavenee WK, editors. Malignant peripheral nerve sheath tumor (MPNST). World Health Organization Classification of Tumours. Pathology and Genetics of Tumours of the Nervous System. Lyon: IARC Press, 2000: 172-174. 